\title{
Fractional impulsive neutral functional differential equations involving $\psi$-Caputo fractional derivative
}

\author{
Shabna.M.S ${ }^{1 *}$ and Ranjini.M.C ${ }^{2}$
}

\begin{abstract}
In this paper, the initial value problem is discussed for a class of fractional impulsive neutral functional differential equation of a function with respect to another function and the criteria on existence and uniqueness are obtained by using known fixed point theorems.
\end{abstract}

Keywords

Fractional order differential equations, fractional impulsive conditions, $\psi$-Caputo fractional derivative.

AMS Subject Classification

26A33, 34A12, 34A34, 34A37, 34K40, 37C25.

${ }_{1,2} P G$ and Research Department of Mathematics, MES Mampad College, Malappuram, Kerala-676542, India.

*Corresponding author: ${ }^{1}$ shabnasaidh@gmail.com; ${ }^{2}$ ranjiniprasad@gmail.com

Article History: Received 24 January 2019; Accepted 24 May 2019

\section{Contents}

1 Introduction .............................. 493

2 Preliminaries ............................. 494

3 Main Results ........................... 495

4 Conclusion ................................ 499

References $\ldots \ldots \ldots \ldots \ldots \ldots \ldots \ldots \ldots \ldots \ldots \ldots, 499$

\section{Introduction}

The aim of this paper is to investigate the existence and uniqueness of solutions for fractional Impulsive neutral functional differential equation involving the Caputo fractional derivative of a function $x$ with respect to another function $\psi$.

$$
\left\{\begin{array}{l}
{ }^{C} D_{t_{0}}^{\alpha, \psi}\left(x(t)-g\left(t, x_{t}\right)\right)=f\left(t, x_{t}\right), t \in\left(t_{0}, \infty\right), t_{0} \geq 0, t \neq t_{k} \\
\Delta x\left(t_{k}\right)=I_{k}\left(x\left(t_{k}-\right)\right) \\
x_{t_{0}}=\phi
\end{array}\right.
$$

where ${ }^{C} D^{\alpha, \psi}$ is the Caputo fractional derivative of order $0<\alpha<1$ with respect to another function $\psi$.

$f, g:\left(\left[t_{0}, \infty\right) \times C\left([-r, 0], \mathbb{R}^{n}\right)\right) \rightarrow \mathbb{R}^{n}$ are given functions satisfying certain assumptions, which will be specified later, $a>0$ and $\phi \in C\left([-r, 0], \mathbb{R}^{n}\right)$. If $x \in C\left(\left[t_{0}-r, t_{0}+a\right], \mathbb{R}^{n}\right)$, then for any $t \in\left[t_{0}, t_{0}+a\right]$, define $x_{t}$ by $x_{t}(\theta)=x(t+\theta)$ for $\theta \in[-r, 0]$. Let $\psi \in C^{1}\left[t_{0}, \infty\right)$ be a continuous increasing function such that $\psi^{\prime}(x) \neq 0, \forall x \in\left[t_{0}, \infty\right)$.
Let $I_{k}: \mathbb{R}^{n} \rightarrow \mathbb{R}^{n}, \Delta x\left(t_{k}\right)=x\left(t_{k}^{+}\right)-x\left(t_{k}^{-}\right)$with

$x\left(t_{k}^{+}\right)=\lim _{h \rightarrow 0^{+}} x\left(t_{k}+h\right)$,

$x\left(t_{k}^{-}\right)=\lim _{h \rightarrow 0^{-}} x\left(t_{k}-h\right), k=1,2, \cdots, m$

for $t_{0}<t_{1}<t_{2}<\cdots<t_{m}$.

Fractional calculus is a branch of mathematical analysis that studies the several different properties of defining real number powers or complex number powers of the differentiation operator and the integration operator. The concept of fractional derivative appeared for the first time in a famous correspondence between G.A de L'Hospital and G.W. Leibniz, in 1695[2]. Fractional calculus is a branch of Mathematics, where in the differential equations, ordinary and partial derivatives are replaced by Fractional operators. For more than two centuries this subject was relevant only in pure mathematics, and Euler, Fourier, Abel, Liouville, Riemann, Hadamard, among others, have studied fractional operators, by presenting new definitions and studying their most important properties. Nowadays Fractional calculus has tremendous applications. Viscoelasticity, electrical circuits, electro magnetism, sound propagation, Fluid Mechanics, edge detection, lateral and longitudinal control, Cardiac tissue electrode interface, Earth system dynamics are some of them[12-14]. Many authors discussed about Neutral differential equations, as it have importance in many areas of applied Mathematics[3-5]. Ricardo Almeida has many studies on the topic Fractional derivative of function with respect to another function, which will be very useful in applied mathematics[6, 7, 9].

Motivated by the paper of Ricardo Almeida[6, 7, 9] and [3], we started studying about fractional differential equation 
of function with respect to another function and fractional Impulsive neutral functional differential equations. Through this paper we are discussing the initial Value Problem for a class of fractional neutral functional differential equations with bounded delay of a function with respect to another function. In the preliminary section we present essential definitions and results. Then based on many assumptions and Krasnoselskii's fixed point theorem, we prove that $I V P(1.1)$ has at least one solution, by deducing $\operatorname{IVP}(1.1)$ into equivalent integral equation. Also we prove the uniqueness of the solution using Banach fixed point theorem.

We know that there are plenty of Fractional operators, $\psi$-Caputo fractional operators are general operators, from which choosing special kernels and different differential operators, we can obtain classical fractional derivatives and integrals[6]. For example to get the Riemann-Liouville fractional derivative, it enough to take the kernel as $k(x, t)=x-t$ and differential operator as $d / d x$. Choosing $k(x, t)=\ln (x / t)$ and differential $x d / d x$ we obtain Hadamard fractional derivative. Throughout our study we are considering kernel as $k(x, t)=\psi(x)-\psi(t)$ and derivative operator as $1 / \psi^{\prime}(x) d / d x$. If we consider $\psi(x)=x$ or $\psi(x)=\ln (x)$, we obtain RiemannLiouville and Hadamard Fractional derivatives respectively. Existence and uniqueness results for boundary value problem with mixed boundary conditions and initial value problem of nonlinear fractional differential equations involving Caputotype fractional derivative with respect to another function were already discussed [7, 9]

\section{Preliminaries}

In this section we introduce some definitions and preliminary facts which are used through the paper. Let $J \subset \mathbb{R}$, define $C\left(J, \mathbb{R}^{n}\right)$ be the Banach space of all continuous functions from $J$ to $\mathbb{R}^{n}$ with the norm

$$
\|x\|=\sup _{t \in J}|x(t)|
$$

where $|\cdot|$ denotes a suitable norm on $\mathbb{R}^{n}$

Also consider the Banach space $P C\left(J, \mathbb{R}^{n}\right)=\left\{x: J \rightarrow \mathbb{R}^{n}\right.$ : $x \in C\left(\left(t_{k}, t_{k+1}\right], \mathbb{R}^{n}\right), k=0,1, \cdots, m$ and there exist $x\left(t_{k}^{+}\right)$and $x\left(t_{k}^{-}\right), k=1,2, \cdots, m$ with $\left.x\left(t_{k}^{-}\right)=x(k)\right\}$

Definition 2.1. [1, 3] The Riemann-Liouville fractional integral of order $\alpha$ with lower limit $t_{0}$ for a function $f$ is defined as

$$
I^{\alpha} f(t)=\frac{1}{\Gamma(\alpha)} \int_{t_{0}}^{t} \frac{f(s)}{(t-s)^{1-\alpha}} d s, \quad t>t_{0}, \quad \alpha>0
$$

Definition 2.2. [1, 3] Riemann-Liouville derivative of order $\alpha$ with lower limit $t_{0}$ for a function $f:\left[t_{0}, \infty\right) \longrightarrow \mathbb{R}$ can be written as

$$
\begin{array}{r}
D^{\alpha} f(t)=\frac{1}{\Gamma(n-\alpha)} \frac{d^{n}}{d x^{n}} \int_{t_{0}}^{t} \frac{f(s)}{(t-s)^{\alpha+1-n}} d s, \\
t>t_{0}, \quad n-1<\alpha<n
\end{array}
$$

Definition 2.3. [1, 3] Caputo derivative of order $\alpha$ with the lower limit $t_{0}$ for a function

$f:\left[t_{0}, \infty\right) \rightarrow \mathbb{R}$ can be written as

$$
\begin{array}{r}
{ }^{C} D^{\alpha} f(t)=\frac{1}{\Gamma(n-\alpha)} \int_{t_{0}}^{t} \frac{f^{(n)}(s)}{(t-s)^{\alpha+1-n}} d s=I^{n-\alpha} f^{(n)}(t), \\
t>t_{0}, n-1<\alpha<n
\end{array}
$$

Obviously Caputo derivative of a constant is equal to zero.

Here we deal with fractional derivatives and fractional integrals with respect to another function.

Definition 2.4. $[1,6]$ Let $\alpha>0, I=[a, b]$ be a finite or infinite interval, $f$ an integrable function defined on $I$ and $\psi \in C^{1}(I)$, an increasing function such that $\psi^{\prime}(x) \neq 0, \forall x \in I$. Fractional integrals and fractional derivatives of a function $f$ with respect to another function $\psi$ are defined as

$$
I_{a}^{\alpha, \psi} f(t)=\frac{1}{\Gamma(\alpha)} \int_{a}^{t} \psi^{\prime}(s)[\psi(t)-\psi(s)]^{\alpha-1} f(s) d s
$$

and

$$
\begin{gathered}
D_{a}^{\alpha, \psi} f(t)=\left[\frac{1}{\psi^{\prime}(t)} \frac{d}{d t}\right]^{n} I_{a+}^{n-\alpha, \psi} f(t) \\
=\frac{1}{\Gamma(n-\alpha)}\left[\frac{1}{\psi^{\prime}(t)} \frac{d}{d t}\right]^{n} \int_{a}^{t} \psi^{\prime}(s)[\psi(t)-\psi(s)]^{n-\alpha-1} f(s) d s \\
\text { where } n=[\alpha]+1
\end{gathered}
$$

Considering fractional derivatives and fractional integrals with respect to another function $\psi$ and for different choices for $\psi$ we can obtain e.g, the Riemann Lioville, the Hadamard and the Erdélyi-Kober fractional derivatives and fractional integrals. For different kernels and differential operators we obtain different operators.

Definition 2.5. $[6,7,9]$ Let $\alpha>0, n \in \mathbb{N}, I$ is the interval $-\infty \leq a<b \leq \infty, f, \psi \in C^{(n)}(I)$ be two functions such that $\psi$ is increasing and $\psi^{\prime}(t) \neq 0 \quad \forall x \in I$. Then $\psi-$ Caputo fractional derivative of $f$ of order $\alpha$ is given by

$$
{ }^{C} D^{\alpha, \psi} f(t)=I_{a}^{n-\alpha, \psi}\left[\frac{1}{\psi^{\prime}(t)} \frac{d}{d t}\right]^{n} f(t)
$$

where $n=[\alpha]+1$ for $\alpha \notin \mathbb{N}$ and $n=\alpha$ for $\alpha \in \mathbb{N}$

To simplify the notation, we are using the abbreviated symbol

$$
f_{\psi}^{[n]} f(t)=\left[\frac{1}{\psi^{\prime}(t)} \frac{d}{d t}\right]^{n} f(t)
$$

From the definition it is clear that, given $\alpha=m \in \mathbb{N}$,

$$
{ }^{C} D_{a}^{\alpha, \psi} f(t)=f_{\psi}^{[m]}(t)
$$


and if $\alpha \notin \mathbb{N}$, then

${ }^{C} D_{a}^{\alpha, \psi} f(t)=\frac{1}{\Gamma(n-\alpha)} \int_{a}^{t} \psi^{\prime}(s)[\psi(t)-\psi(s)]^{n-\alpha-1} f_{\psi}^{[n]}(s) d s$

In particular if $0<\alpha<1$

$$
{ }^{C} D_{a}^{\alpha, \psi} f(t)=\frac{1}{\Gamma(1-\alpha)} \int_{a}^{t}[\psi(t)-\psi(s)]^{-\alpha} f^{\prime}(s) d s
$$

Remark 2.6. $[6,7,9]$ Given a function $f \in C^{n}[a, b]$ and $\alpha>0$

we have

$$
{ }^{C} D_{a}^{\alpha, \psi} f(t)=D_{a}^{\alpha, \psi}\left[f(t)-\sum_{k=0}^{n-1} \frac{f_{\psi}^{[k]}(a)}{k !}[\psi(t)-\psi(a)]^{k}\right]
$$

where $n=[\alpha]+1$

$$
I_{a}^{\alpha, \psi} f(t)=f(t)-\sum_{k=0}^{n-1} \frac{f_{\psi}^{[k]}(a)}{k !}[\psi(x)-\psi(a)]^{k}
$$

In particular if $0<\alpha<1$, we have

$$
I_{a}^{\alpha, \psi C} D_{a}^{\alpha, \psi} f(t)=f(t)-f(a)
$$

Also.

$$
{ }^{C} D_{a}^{\alpha, \psi} I_{a}^{\alpha, \psi} f(t)=f(t)
$$

Lemma 2.7. (Kranoselskii's Fixed Point Theorem) Let $X$ be a Banach space, let $E$ be a bounded closed convex subset of $X$ and let $S, U$ be maps of $E$ into $X$ such that $S x+$ $U y \in E$ for every pair $x, y \in E$. If $S$ is a contraction and $U$ is completely continuous, then the equation $S x+U x=x$ has a solution on $E$.

Note that

$$
\begin{aligned}
x(t)=x_{0} & -\frac{1}{\Gamma(\alpha)} \int_{0}^{a} \psi^{\prime}(s)(\psi(a)-\psi(s))^{\alpha-1} h(s) d s \\
& +\frac{1}{\Gamma(\alpha)} \int_{0}^{t} \psi^{\prime}(s)(\psi(t)-\psi(s))^{\alpha-1} h(s) d s
\end{aligned}
$$

solves the Cauchy Problems

$$
\left\{\begin{array}{l}
{ }^{C} D^{\alpha, \psi} x(t)=h(t), \quad t \in J=[0, T], T \geq 0 \\
x(0)=x_{0}-\frac{1}{\Gamma(\alpha)} \int_{0}^{a} \psi^{\prime}(s)(\psi(a)-\psi(s))^{\alpha-1} h(s) d s
\end{array}\right.
$$

One can obtain the following result immediately

Lemma 2.8. Let $\alpha \in(0,1)$ and $h: J \rightarrow \mathbb{R}$ be continuous. A function $x \in C(J, \mathbb{R})$ is a solution of the fractional integral equation

$$
\begin{aligned}
x(t)=x_{0} & -\frac{1}{\Gamma(\alpha)} \int_{0}^{a} \psi^{\prime}(s)(\psi(a)-\psi(s))^{\alpha-1} h(s) d s \\
& +\frac{1}{\Gamma(\alpha)} \int_{0}^{t} \psi^{\prime}(s)(\psi(t)-\psi(s))^{\alpha-1} h(s) d s
\end{aligned}
$$

if and only if $x$ is a solution of the following fractional Cauchy problems

$$
\begin{cases}{ }^{C} D^{\alpha, \psi} x(t) & =h(t), \quad t \in J \\ x(a) & =x_{0}, \quad a>0\end{cases}
$$

\section{Main Results}

Let $I_{0}=\left[t_{0}, t_{0}+\delta\right]$

$$
\begin{array}{r}
A(\delta, \gamma)=\left\{x \in C\left(\left[t_{0}-r, t_{0}+\delta\right], \mathbb{R}^{n}\right) \mid x_{t_{0}}=\phi,\right. \\
\left.\sup _{t_{0} \leq t \leq t_{0}+\delta}|x(t)-\phi(0)| \leq \gamma\right\}
\end{array}
$$

where $\delta, \gamma$ are positive constants. Before starting and proving the main results, we introduce the following hypotheses.

(H1) $f(t, \phi)$ is measurable with respect to $t$ on $I_{0}$.

(H2) $f(t, \phi)$ is continuous with respect to $\phi$ on $C\left([-r, 0], \mathbb{R}^{n}\right)$.

(H3) There exist $\alpha_{1} \in(0, \alpha)$ and a real valued function $m(t) \in$ $L^{\frac{1}{\alpha_{1}}}\left(I_{0}\right)$ such that for any $x \in A(\delta, \gamma),\left|f\left(t, x_{t}\right)\right| \leq m(t)$, $M=\|m\|_{L^{\frac{1}{\alpha_{1}}}\left(I_{0}\right)}$ for $t \in I_{0}$.

(H4) For any $x \in A(\delta, \gamma), g\left(t, x_{t}\right)=g_{1}\left(t, x_{t}\right)+g_{2}\left(t, x_{t}\right)$.

(H5) $g_{1}$ is continuous and for any $x^{\prime}, x^{\prime \prime} \in A(\delta, \gamma), t \in I_{0}$, $\left|g_{1}\left(t, x_{t}^{\prime}\right)-g_{1}\left(t, x_{t}^{\prime \prime}\right)\right| \leq l|| x^{\prime}-x^{\prime \prime}||$, where $l \in(0,1)$

(H6) $g_{2}$ is completely continuous and for any bounded set $\Lambda$ in $A(\delta, \gamma)$,

the set $\left\{t \rightarrow g_{2}\left(t, x_{t}\right): x \in \Lambda\right\}$ is equicontinuous in $C\left(I_{0}, \mathbb{R}^{n}\right)$.

(H7) The functions $I_{k}: \mathbb{R}^{n} \rightarrow \mathbb{R}^{n}$ are continuous and there exist a constant $0<L<1$ such that $\left\|I_{k}(x)-I_{k}(y)\right\| \leq \frac{L}{m}\|x-y\|, x, y \in \mathbb{R}^{n}$, $m>0, k=1,2, \cdots, m$.

Lemma 3.1. If there exist $\delta \in(0, a)$ and $\gamma \in(0, \infty)$ such that $(H 1)-(H 3)$ are satisfied, then for $t \in\left(t_{0}, t_{0}+\delta\right], I V P(1.1)$ is equivalent to the following equation.

$x(t)=$

$$
\left\{\begin{array}{r}
\phi(0)-g\left(t_{0}, \phi\right)+g\left(t, x_{t}\right)+I_{t_{0}}^{\alpha, \psi} f\left(t, x_{t}\right), t \in\left[t_{0}, t_{1}\right] \\
\phi(0)-g\left(t_{0}, \phi\right)+g\left(t, x_{t}\right)+I_{1}\left(x\left(t_{1}-\right)\right)+I_{t_{0}}^{\alpha, \psi} f\left(t, x_{t}\right), \\
t \in\left(t_{1}, t_{2}\right] \\
\phi(0)-g\left(t_{0}, \phi\right)+g\left(t, x_{t}\right)+I_{1}\left(x\left(t_{1}-\right)\right)+I_{2}\left(x\left(t_{2}-\right)\right) \\
\quad+I_{t_{0}}^{\alpha, \psi} f\left(t, x_{t}\right), t \in\left(t_{2}, t_{3}\right] \\
\begin{array}{r}
\vdots \\
\phi(0)-g\left(t_{0}, \phi\right)+g\left(t, x_{t}\right)+\sum_{t_{0}<t<t_{k}} I_{i}\left(x\left(t_{i}-\right)\right) \\
\\
\quad+I_{t_{0}}^{\alpha, \psi} f\left(t, x_{t}\right), t \in\left(t_{m}, t_{0}+\delta\right]
\end{array}
\end{array}\right.
$$

$x_{t_{0}}=\phi$ 
Proof. Assume $x$ satisfies $I V P(1.1)$.

If $t \in\left[t_{0}, t_{1}\right]$, then ${ }^{C} D_{t_{0}}^{\alpha, \psi}\left(x(t)-g\left(t, x_{t}\right)\right)=f\left(t, x_{t}\right), t \in\left(t_{0}, t_{1}\right]$. Integrating from $t_{0}$ to $t$ by virtue of definition $(2.5)$, we can obtain

$$
x(t)=\phi(0)-g\left(t_{0}, \phi\right)+g\left(t, x_{t}\right)+I_{t_{0}}^{\alpha, \psi} f\left(t, x_{t}\right)
$$

If $t \in\left(t_{1}, t_{2}\right]$,

then ${ }^{C} D_{t_{0}}^{\alpha, \psi}\left(x(t)-g\left(t, x_{t}\right)\right)=f\left(t, x_{t}\right), t \in\left(t_{1}, t_{2}\right]$ with $x\left(t_{1}+\right)=x\left(t_{1}-\right)+I_{1}\left(u\left(t_{1}-\right)\right)$

By lemma (2.8), one obtain

$$
\begin{aligned}
& x(t)=x\left(t_{1}+\right)-g\left(t_{1}, x_{t_{1}}\right)+g\left(t, x_{t}\right) \\
& -\frac{1}{\Gamma(\alpha)} \int_{t_{0}}^{t_{1}} \psi^{\prime}(s)\left(\psi\left(t_{1}\right)-\psi(s)\right)^{\alpha-1} f\left(s, x_{s}\right) d s \\
& +I_{t_{0}}^{\alpha, \psi} f\left(t, x_{t}\right) \\
& =x\left(t_{1}-\right)+I_{1}\left(x\left(t_{1}-\right)\right)-g\left(t_{1}, x_{t_{1}}\right) \\
& -\frac{1}{\Gamma(\alpha)} \int_{t_{0}}^{t_{1}} \psi^{\prime}(s)\left(\psi\left(t_{1}\right)-\psi(s)\right)^{\alpha-1} f\left(s, x_{s}\right) d s \\
& +I_{t_{0}}^{\alpha, \psi} f\left(t, x_{t}\right) \\
& \\
& +I_{1}(t)=\phi(0)-g\left(t_{0}, \phi\right)+g\left(t, x_{t}\right) \\
& +I_{t_{0}}^{\alpha, \psi} f\left(t, x_{t}\right) \\
& =x(0)-g\left(t_{0}, \phi\right)+g\left(t, x_{t}\right)+I_{1}\left(x\left(t_{1}-\right)+I_{t_{0}}^{\alpha, \psi} f\left(t, x_{t}\right)\right. \\
& +I_{t_{0}}^{\alpha, \psi} f\left(t, x_{t}\right) \\
& \\
& \\
& \\
& \\
&
\end{aligned}
$$

If $t \in\left(t_{m}, t_{0}+\delta\right]$, then again by lemma (2.8), we get $x(t)=\phi(0)-g\left(t_{0}, \phi\right)+g\left(t, x_{t}\right)+\sum_{i=1}^{m} I_{i}\left(x\left(t_{i}-\right)+I_{t_{0}}^{\alpha, \psi} f\left(t, x_{t}\right)\right.$

Conversely, assume that $x$ satisfies equation (6). If $t \in$ $\left(t_{0}, t_{1}\right]$ and using equation( 3$)$, we get ${ }^{C} D_{t_{0}}^{\alpha, \psi}\left(x(t)-g\left(t, x_{t}\right)=f\left(t, x_{t}\right)\right.$.

If $t \in\left(t_{k}, t_{k+1}\right], k=1,2, \cdots, m$ and using the fact of $\psi-$ Caputo fractional derivative of a constant is equal to zero, we obtain ${ }^{C} D_{t_{0}}^{\alpha, \psi}\left(x(t)-g\left(t, x_{t}\right)\right)=f\left(t, x_{t}\right), t \in\left(t_{k-1}, t_{k}\right]$ and $u\left(t_{k}+\right)-u\left(t_{k}-\right)=I_{k}\left(u\left(t_{k}-\right)\right), k=1,2, \cdots, m$

This completes the proof.

Theorem 3.2. Assume that there exist $\delta \in(0, a)$ and $\gamma \in$ $(0, \infty)$ such that $(H 1)-(H 7)$ are satisfied, then IVP $(1.1)$ has at least one solution

$$
\begin{cases}x(t) & =\phi(0)-g\left(t_{0}, \phi\right)+g\left(t, x_{t}\right)+\sum_{t_{0}<t_{k}<t} I_{k}\left(x\left(t_{k}-\right)\right) \\ & +\frac{1}{\Gamma(\alpha)} \int_{t_{0}}^{t} \psi^{\prime}(s)[\psi(t)-\psi(s)]^{\alpha-1} f\left(s, x_{s}\right) d s \\ x_{t_{0}}=\phi & \end{cases}
$$

on $\left[t_{0}, t_{0}+\eta\right]$ for some positive number $\eta$.

Proof. According to (H4), equation (3.1) is equivalent to the following equation

$$
\begin{cases}x(t) \quad & =\phi(0)-g_{1}\left(t_{0}, \phi\right)-g_{2}\left(t_{0}, \phi\right) \\ & +g_{1}\left(t, x_{t}\right)+g_{2}\left(t, x_{t}\right)+\Sigma_{t_{0}<t_{k}<t} I_{k}\left(x\left(t_{k}-\right)\right) \\ & +\frac{1}{\Gamma(\alpha)} \int_{t_{0}}^{t} \psi^{\prime}(s)[\psi(t)-\psi(s)]^{\alpha-1} f\left(s, x_{s}\right) d s \\ & t \in I_{0} \\ x_{t_{0}}=\phi & \end{cases}
$$

Let $\tilde{\phi} \in A(\delta, \gamma)$ be defined as

$$
\tilde{\phi}_{t_{0}}=\phi, \quad \tilde{\phi}\left(t_{0}+t\right)=\phi(0) \quad \forall t \in[0, \delta] .
$$

If $x$ is a solution of the $\operatorname{IVP}(1.1)$, let $x\left(t_{0}+t\right)=\tilde{\phi}\left(t_{0}+t\right)+y(t), \quad t \in[-r, \delta]$ Then we have, $\quad x_{t_{0}+t}=\tilde{\phi}_{t_{0}+t}+y_{t}, \quad t \in[0, \delta]$. Thus

$$
\begin{array}{r}
y(t)=-g_{1}\left(t_{0}, \phi\right)-g_{2}\left(t_{0}, \phi\right)+g_{1}\left(t_{0}+t, y_{t}+\tilde{\phi}_{t_{0}+t}\right) \\
+g_{2}\left(t_{0}+t, y_{t}+\tilde{\phi}_{t_{0}+t}\right)+\sum_{t_{0}<t_{k}<t} I_{k}\left(x\left(t_{k}-\right)\right) \\
+\frac{1}{\Gamma(\alpha)} \int_{0}^{t} \psi^{\prime}\left(s+t_{0}\right)\left[\psi\left(t+t_{0}\right)-\psi\left(s+t_{0}\right)\right]^{\alpha-1} \\
f\left(t_{0}+s, y_{s}+\tilde{\phi}_{t_{0}+s}\right) d s, t \in[0, \delta]
\end{array}
$$

Since $g_{1}, g_{2}$ are continuous and $x_{t}$ is continuous in $\mathrm{t}$, there exist $\delta^{\prime}>0$ when $0<t<\delta^{\prime}$

$$
\left|g_{1}\left(t_{0}+t, y_{t}+\tilde{\phi}_{t_{0}+t}\right)-g_{1}\left(t_{0}, \phi\right)\right|<\frac{\gamma}{4}
$$




$$
\left|g_{2}\left(t_{0}+t, y_{t}+\tilde{\phi}_{t_{0}+t}\right)-g_{2}\left(t_{0}, \phi\right)\right|<\frac{\gamma}{4}
$$

Choose

$$
\eta=\min \left\{\delta, \delta^{\prime},\left(\frac{\gamma \Gamma(\alpha+1)}{4 M}\right)^{1 / \alpha}\right\}
$$

Define $E(\eta, \gamma)$ as follows

$$
\begin{array}{r}
E(\eta, \gamma)=\left\{y \in P C\left([-r, \eta], \mathbb{R}^{n}\right) / y(s)=0\right. \\
\text { for } s \in[-r, 0] \text { and }\|y\| \leq r\} .
\end{array}
$$

Then $E(\eta, \gamma)$ is a closed bounded and convex subset of $P C\left([-r, \eta], \mathbb{R}^{n}\right)$.

On $E(\eta, \gamma)$, we define the operators $S$ and $U$ as follows

$$
\begin{gathered}
S y(t)= \begin{cases}0 & \text { if } t \in[-r, 0] \\
-g_{1}\left(t_{0}, \phi\right)+g_{1}\left(t_{0}+t, y_{t}+\tilde{\phi}_{t_{0}+t}\right) & \text { if } t \in[0, \eta]\end{cases} \\
U y(t)= \begin{cases}0 & \text { if } t \in[-r, 0] \\
-g_{2}\left(t_{0}, \phi\right)+g_{2}\left(t_{0}+t, y_{t}+\tilde{\phi}_{t_{0}+t}\right) & \\
+\sum_{t_{0}<t_{k}<t} I_{k}\left(x\left(t_{k}-\right)\right) & \frac{1}{\Gamma(\alpha)} \int_{0}^{t} \psi^{\prime}\left(s+t_{0}\right)\left(\psi\left(t+t_{0}\right)-\psi\left(s+t_{0}\right)\right)^{\alpha-1} \\
f\left(t_{0}+s, y_{s}+\tilde{\phi}_{t_{0}+s}\right) d s & \text { if } t \in[0, \eta]\end{cases}
\end{gathered}
$$

It is easy to see that if the operator equation

$$
y=S y+U y
$$

has a solution $y \in E(\eta, \gamma)$ if and only if $y$ is a solution of (3.3).

Thus $x\left(t_{0}+t\right)=y(t)+\tilde{\phi}\left(t_{0}+t\right)$ is a solution of $I V P(1.1)$ on $[0, \eta]$. Therefore the existence of a solution of the IVP(1.1) is equivalent that equation (3.7) has a fixed point in $E(\eta, \gamma)$. Now we show that $S+U$ has a fixed point in $E(\eta, \gamma)$. The proof is divided in to three steps.

Step I: $S z+U y \in E(\eta, \gamma)$ for every pair $z, y \in E(\eta, \gamma)$. In fact, for every pair $z, y \in E(\eta, \gamma), S z+U y \in P C\left([-r, \eta], \mathbb{R}^{n}\right)$. Also it is obvious that $(S z+U y)(t)=0, t \in[-r, 0]$.

Moreover, for $t \in[0, \eta]$, by (3.4), (3.5), (3.6) and the condition $(H 3)$ and if $\left(\psi\left(t+t_{0}\right)-\psi\left(t_{0}\right)\right)<\delta$, we have,

$$
\begin{gathered}
|S z(t)-U y(t)| \leq\left|-g_{1}\left(t_{0}, \phi\right)+g_{1}\left(t_{0}+t, z_{t}+\tilde{\phi}_{t_{0}+t}\right)\right| \\
+\left|-g_{2}\left(t_{0}, \phi\right)+g_{2}\left(t_{0}+t, y_{t}+\tilde{\phi}_{t_{0}+t}\right)\right| \\
\quad+\sum_{t_{0}<t_{k}<t}\left|I_{k}\left(x\left(t_{k}-\right)\right)\right| \\
+\frac{1}{\Gamma(\alpha)} \int_{0}^{t} \mid \psi^{\prime}\left(s+t_{0}\right)\left[\psi\left(t+t_{0}\right)-\psi\left(s+t_{0}\right)\right]^{\alpha-1} \\
\quad f\left(t_{0}+s, y_{s}+\tilde{\phi}_{t_{0}+s}\right) \mid d s \\
\leq \frac{\gamma}{2}+\frac{M}{\Gamma(\alpha+1)}\left(\psi\left(t+t_{0}\right)-\psi\left(t_{0}\right)\right)^{\alpha}+L \gamma \\
\leq \frac{\gamma}{2}+\frac{M}{\Gamma(\alpha+1)} \eta^{\alpha}+L \gamma \\
\leq \gamma
\end{gathered}
$$

Therefore

$$
|| S z+U y||=\sup _{t \in[0, \eta]}|(S z)(t)+(U y)(t)| \leq \gamma,
$$

which means that $S z+U y \in E(\eta, \gamma)$, for any $z, y \in E(\eta, \gamma)$.

Step II: $S$ is a contraction on $E(\eta, \gamma)$.

For any $y^{\prime}, y^{\prime \prime} \in E(\eta, \gamma), y_{t}^{\prime}+\tilde{\phi}_{t_{0}+t}, y_{t}^{\prime \prime}+\tilde{\phi}_{t_{0}+t} \in A(\delta, \gamma)$.

Also by (H5), we get that

$$
\begin{gathered}
\left|S y^{\prime}(t)-S y^{\prime \prime}(t)\right| \\
=\left|g_{1}\left(t_{0}+t, y_{t}^{\prime}+\tilde{\phi}_{t_{0}+t}\right)-g_{1}\left(t_{0}+t, y_{t}^{\prime \prime}+\tilde{\phi}_{t_{0}+t}\right)\right| \\
\leq l|| y^{\prime}-y^{\prime \prime}||
\end{gathered}
$$

which implies that

$$
\left\|S y^{\prime}-S y^{\prime \prime}|| \leq l\right\| y^{\prime}-y^{\prime \prime} \|
$$

In view of $0<l<1, S$ is a contraction on $E(\eta, \gamma)$.

Step III: Now we show that $U$ is a completely continuous operator.

$$
U_{1} y(t)=\left\{\begin{array}{lr}
0 & t \in[-r, 0], \\
-g_{2}\left(t_{0}, \phi\right)+g_{2}\left(t_{0}+t, y_{t}+\tilde{\phi}_{t_{0}+t}\right) & t \in[0, \eta] .
\end{array}\right.
$$

and

$$
U_{2} y(t)=\left\{\begin{array}{lc}
0 & t \in[-r, 0] \\
\sum_{t_{0}<t_{k}<t} I_{k}\left(x\left(t_{k}-\right)\right)+ & \frac{1}{\Gamma(\alpha)} \int_{0}^{t} \psi^{\prime}\left(s+t_{0}\right)\left[\psi\left(t+t_{0}\right)-\psi\left(s+t_{0}\right)\right]^{\alpha-1} \\
& f\left(t_{0}+s, y_{s}+\tilde{\phi}_{t_{0}+s}\right) d s t \in[0, \eta]
\end{array}\right.
$$

Clearly $U=U_{1}+U_{2}$ 
Since $g_{2}$ is completely continuous, $U_{1}$ is continuous and $\left\{U_{1} y: y \in E(\eta, \gamma)\right\}$ is uniformly bounded, from the condition that the set $\left\{t \rightarrow g_{2}\left(t, x_{t}\right): x \in \Lambda\right\}$ be equicontinuous for any bounded set $\Lambda$ in $A(\delta, \gamma)$, we can conclude that $U_{1}$ is a completely continuous operator.

On the other hand for any $t \in[0, \eta]$, we have

$$
\begin{aligned}
& \left\|U_{2} y(t)\right\| \leq \sum_{t_{0}<t_{k}<t}\left|I_{i}\left(x\left(t_{i}-\right)\right)\right| \\
& +\frac{1}{\Gamma(\alpha)} \int_{0}^{t} \mid \psi^{\prime}\left(s+t_{0}\right)\left[\psi\left(t+t_{0}\right)-\psi\left(s+t_{0}\right)\right]^{\alpha-1} \\
& \quad f\left(t_{0}+s, y_{s}+\tilde{\phi}_{t_{0}+s}\right) \mid d s \\
& \leq L \gamma+\left(\psi\left(t+t_{0}\right)-\psi\left(t_{0}\right)^{\alpha} \frac{M}{\Gamma(\alpha+1)}\right. \\
& \leq L \gamma+\eta^{\alpha} \frac{M}{\Gamma(\alpha)}
\end{aligned}
$$

Hence $\left\{U_{2}(y): y \in E(\eta, \gamma)\right\}$ is uniformly bounded.

Now we will prove that $\left\{U_{2} y: y \in E(\eta, \gamma)\right\}$ is equicontinuous. For any $0 \leq t_{1}<t_{2} \leq \eta$ and $y \in E(\eta, \gamma)$,

and if $|\psi(x)-\psi(y)| \leq N|| x-y||, 0<N<1 \quad \forall x, y \in[0, \eta]$ we get that,

$$
\begin{aligned}
& \left|U_{2} y\left(t_{2}\right)-U_{2} y\left(t_{1}\right)\right| \leq \\
& \frac{1}{\Gamma(\alpha)} \int_{0}^{t_{2}} \mid \psi^{\prime}\left(s+t_{0}\right)\left[\left(\psi\left(t_{2}+t_{0}\right)-\psi\left(s+t_{0}\right)\right)^{\alpha-1}\right] \\
& f\left(t_{0}+s, y_{s}+\tilde{\phi}_{\left.t_{0}+s\right)} \mid d s\right. \\
& +\frac{1}{\Gamma(\alpha)} \int_{0}^{t_{1}} \mid \psi^{\prime}\left(s+t_{0}\right)\left[\psi\left(t_{1}+t_{0}\right)-\psi\left(s+t_{0}\right)\right]^{\alpha-1} \\
& f\left(t_{0}+s, y_{s}+\tilde{\phi}_{t_{0}+s}\right) \mid d s \\
& +\sum_{t_{0}<t_{k}<t_{2}-t_{1}} I_{k}\left(x\left(t_{k}-\right)\right) \\
& \leq \frac{M}{\Gamma(\alpha+1)} \\
& {\left[\left(\psi\left(t_{2}+t_{0}\right)-\psi\left(t_{0}\right)\right)^{\alpha}-\left(\psi\left(t_{1}+t_{0}\right)-\psi\left(t_{0}\right)\right)^{\alpha}\right]} \\
& +\sum_{t_{0}<t_{k}<t_{2}-t_{1}} I_{k}\left(x\left(t_{k}-\right)\right)
\end{aligned}
$$

which converges to zero as $t_{1} \rightarrow t_{2}$

Hence $\left\{U_{2} y: y \in E(\eta, \gamma)\right\}$ is equicontinuous. Moreover ,it is clear that $U_{2}$ is continuous. So $U_{2}$ is completely continuous operator. Then $U=U_{1}+U_{2}$ is a completely continuous operator.

Therefore, Krasnoselskii's fixed point theorem shows that $S+U$ has a fixed point on $E(\eta, \gamma)$ and hence the $I V P(1.1)$ has a solution $x(t)=\phi(0)+y\left(t-t_{0}\right)$ for all $t \in\left[t_{0}, t_{0}+\eta\right]$. This completes the proof.

In the case where $g_{1} \equiv 0$, we get the following result.

Corollary 3.3. Assume that there exist $\delta \in(0, a)$ and $\gamma \in$ $(0, \infty)$ such that $(H 1)-(H 3)$ hold and $(H 5)^{\prime} g$ is continuous and for any $x^{\prime}, x^{\prime \prime} \in A(\delta, \gamma), t \in I_{0}$.

$$
\left.\mid g\left(t, x_{t}^{\prime}\right)-g\left(t, x_{t}^{\prime \prime}\right)\right)|\leq l|\left|x^{\prime}-x^{\prime \prime}\right| \mid, l \in(0,1)
$$

Then IVP(1.1) has at least one solution on $\left[t_{0}, t_{0}+\eta\right]$ for some positive number $\eta$.

In the case where $g_{2} \equiv 0$, we have the following result

Corollary 3.4. Assume that there exist $\delta \in(0, a)$ and $\gamma \in$ $(0, \infty)$ such that $(H 1)-(H 3)$ hold and $(H 6)^{\prime} g$ is completely continuous and for any bounded set $\Lambda$ in $A(\delta, \gamma)$, the set $\left\{t \rightarrow g\left(t, x_{t}\right): x \in \Lambda\right\}$ is equicontinuous on $C\left(I_{0}, \mathbb{R}^{n}\right)$. Then IVP(1.1) has at least one solution on $\left[t_{0}, t_{0}+\eta\right]$ for some positive number $\eta$.

Theorem 3.5. Assume that the function $f$ is Lipschitz continuous with respect to the second variable. i.e, there exist a positive constant $L_{1}$ such that $\left\|f\left(t, x_{1_{t}}\right)-f\left(t, x_{2_{t}}\right)\right\| \leq L_{1} \| x_{1}-$ $x_{2}|| \forall t \in[a, b], x_{1}, x_{2} \in C\left(\left[t_{0}-r, t_{0}+a\right)\right)$ with $H 5$ and $H 7$, then there is a constant $h \in \mathbb{R}^{+}$such that there exist a unique solution to the IVP(1.1) on the interval $\left[t_{0}, t_{0}+h\right] \subseteq[a, b]$ if $\left(\frac{L_{1}}{\Gamma(\alpha+1)}\left(\psi\left(t_{0}+h\right)-\psi\left(t_{0}\right)\right)^{\alpha}+L+l\right)<1$.

Proof. Define the function $F$ by $F(x, t)$ by

$$
\begin{gathered}
F(x, t) \\
=\phi(0)-g\left(t_{0}, \phi\right)+g\left(t, x_{t}\right)+\sum_{t_{0}<t<t_{k}} I_{i}\left(x\left(t_{i}-\right)\right)+I_{t_{0}}^{\alpha, \psi} f\left(t, x_{t}\right) .
\end{gathered}
$$

Let $U=\left\{x \in C\left(\left[t_{0}-r, t_{0}+a\right], \mathbb{R}^{n}\right):{ }^{C} D_{t_{0}}^{\alpha, \psi} x(t)\right.$ exists and is continuous in $\left.\left[t_{0}, t_{0}+h\right]\right\}$

It is enough to prove that $F: U \rightarrow U$ is a contraction. Let us see that $F$ is well defined. i.e., $F(U) \subseteq U$. Given the function $x \in U$, we see that ${ }_{t_{0}}^{\alpha, \psi}\left(F(x)(t)-g\left(t, x_{t}\right)\right)=f\left(t, x_{t}\right)$ is continuous and

$$
\begin{gathered}
F(x)(t)=\phi(0)+g\left(t_{0}, \phi\right)+g\left(t, x_{t}\right)+ \\
\sum_{t_{0}<t<t_{k}} I_{i}\left(x\left(t_{i}-\right)\right)+I_{t_{0}}^{\alpha, \psi} f\left(t, x_{t}\right),
\end{gathered}
$$

which satisfies the required conditions.

Now let $x_{1}, x_{2} \in U$ be arbitrary, then by assumptions, we have

$$
\begin{aligned}
& \left\|F\left(x_{1}, t\right)-F\left(x_{2}, t\right)\right\| \\
& \begin{array}{l}
\leq\left\|I_{t_{0}}^{\alpha, \psi}\left(f\left(t, x_{1_{t}}\right)-f\left(t, x_{2_{t}}\right)\right)\right\| \\
\quad+\sum_{t_{0}<t<t_{k}}\left\|\left(I_{i}\left(x_{1}\left(t_{i}-\right)\right)-I_{i}\left(x_{1}\left(t_{i}-\right)\right)\right)\right\| \\
\quad+\left\|g\left(t, x_{1_{t}}\right)-g\left(t, x_{2_{t}}\right)\right\| \\
\leq\left[\frac{L_{1}}{(\Gamma(\alpha+1))}\left(\psi\left(t_{0}+h\right)-\psi\left(t_{0}\right)\right)^{\alpha}+L+l\right]\left\|x_{1}-x_{2}\right\|,
\end{array}
\end{aligned}
$$

which proves that $F$ is a contraction. Using the Banach fixed point theorem, $I V P(1.1)$ has a unique solution 


\section{Conclusion}

In this paper, an approach has been developed for some results on existence and uniqueness of solutions for $\psi$-Caputo Neutral fractional differential equation (1.1), with impulsive conditions. In many papers the existence and uniqueness results have been established. $\psi$-Caputo fractional operators are general operators, from which choosing special kernels and some form of differential operators, we obtain classical fractional operators. To prove the existence we used Krasnoselskii's fixed point theorem and Banach fixed point theorem for uniqueness.

\section{References}

[1] A. A. Kilbas, H. M. Srivastava and J. J. Trujillo. Theory and applications of Fractional Differential equations, North-Holland Mathematics Studies, 204, Elsevier Science B. V.,Amsterdam, 2006.

[2] Yong Zhou, JinRong Wang, Lu Zhang. Basic Theory of Fractional Differential Equations, Second Edition,WSPC World Scientific Co. Pte,Ltd (2017).

[3] R. P. Agarwal, Yong Zhou, Yunyun He. Existence of fractional neutral functional differential equations, Computers and Mathematics with Applications, Vol. 59(2010) No. 3, pp 1095-1100. .

[4] W. R. Melvin. A class of Neutral Functional Differential Equations, Journal of Differential Equations, Vol. 12(1972), No. 3, pp 524-534.

[5] Runping Ye, Guowei Zhang. Neutral Functional Differential Equations of Second order with infinite Delays, Electronic Journal of Differential Equations, Vol. 2010(2010), No.36, pp 1-12.

[6] Ricardo Almeida. A Caputo fractional derivative of a function with respect to another function, Communications in Nonlinear Science and Numerical Simulation, Vol. 44(2017), pp 460-481.

[7] Ricardo Almeida, Agnieszka B. Malinowska, M. Teresa T. Monteiro. Fractional differential equations with a $\mathrm{Ca}$ puto derivative with respect to a Kernel function and their applications, Mathematical Methods in the Applied Sciences, WILEY, Vol. 41)(2018), No. 1, pp. 336-352.

[8] J. Vanterler da C. Sousa, E Capelar de Oliveira, On the $\psi$ Hilfer fractional derivative, Communication in Nonlinear Science and Numerical Simulation, Vol. 60(2018), pp 72-91.

[9] Ricardo Almeida, Fractional differential equations with mixed boundary conditions, The Bulletin of the Malaysian Mathematical Society, Series 2(2018).

[10] Krishnan Balachandran,Juan J. Trujillo, The nonlocal Cauchy problem for nonlinear fractional integro differential equations in Banach Spaces, Nonlinear Analysis 72(2010), pp.4587-4593.

[11] William R. Melvin. Some extensions of Krasnoselskii Fixed point theorem, Journal of Differential Equations11,(1972), pp.335-348.
[12] J. A Tenreiro Machado, Manuel F. Silva, Ramiros S. Barbosa, Isabel S. Jesus, Cecilia M Reis, Maria G. Marcos and Alexandra F. Galhano, Some applications of Fractional calculus in Engineering, Mathematical Problems in Engineering, Vol. 2010, Article ID 639801.

[13] Mehdi Dalir, Majid Bashour. Applications of Fractional Calculus, Applied Mathematical Sciences, Vol. 4(2010), No.21, pp.1021-1032.

[14] Yong Zhang, Samantha E. Hansen. A review of applications of fractional calculus in Earth system dynamics, Chaos, Solitons and Fractals, Vol.102(2017), pp.29-46.

[15] JinRong Wang, Yong Zhou,Michal Fečkan. Nonlinear impulsive problems for fractional differential equattions and Ulam stability, Computers and Mathematics with Applications 64 (2012) 3389-3405.

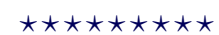

$$
\begin{aligned}
& \operatorname{ISSN}(\mathrm{P}): 2319-3786 \\
& \text { Malaya Journal of Matematik } \\
& \text { ISSN(O):2321 - } 5666
\end{aligned}
$$



